\title{
The Restriction of Qualitative Reasoning Result Based on Rough Set Theory
}

\author{
Han Anyuan \\ Dept. of Equipment Command and Administration, \\ Academy of Armored Force Engineering \\ Beijing, China
}

\author{
Fang Dongxing \\ Ordnance Technology Institute \\ Ordnance Engineering College \\ Shijiazhuang, China
}

Affiliation the Technology Center of Tai Yuan Steel Group CO.

Han Anjing

Taiyuan, China

\begin{abstract}
From the analysis existing problems of Qualitative reasoning results, the necessity of qualitative constraints was put forward. Several existing Qualitative constraints were summed and a new qualitative constraints - rough qualitative constraints was advanced, which uses rough set attribute reduction algorithm, reducing the results of qualitative reasoning. Rough Qualitative reasoning software of the overall design of the process and function modules were introduced. Taking Material Supply Support process to the results of qualitative reasoning as an example, results from the rough qualitative constraint reasoning, reasoning by important results for the material supply security decision-making reference.
\end{abstract}

Keywords-Qualitative constraints, Rough set, Reduction, Reasoning results

\section{I. . THE PROBLEMS IN QUALITATIVE REASONING RESULT}

The qualitative simulation model Based on signed directed graph (Signal Directed Graph, SDG) can express complex causal relationship, with the ability to accommodate large-scale potential of information, particularly for the system dangerous identification and fault diagnosis, has good completeness, but its lower resolution, although the overall result cannot focus. Therefore, the results of reasoning need to go through filtrating, to get useful information. Studying material supply, through analysis of qualitative simulation model of SDG qualitative reasoning results to find the defects aspects in material supply support process, but the various elements of the different results produced by the impact of different factors, the simulation results produce a lot of redundant data[1]. Through filtering it found the important factors, which can provide the basis for decision-making material supply support.

\section{TYPES OF QUALITATIVE CONSTRAINTS}

In early the qualitative constraints including qualitative algebraic constraint and qualitative monotonic relationship between constraints. Qualitative algebraic constraint is some basic algebraic and differential relations, of which "+"," - "," * "," derivative "is a symbol of four basic algebraic constraint, that the qualitative constraints," add, subtract, multiply, except, " qualitative monotonic relationship constraints, that the incremental impact and the reduction between the two constraints[2].
In the process of Qualitative modeling, causality is often defined as the property value of model boundary, some heuristic rules are often used as the conditions of a model deflection (deflection is defined as the static model of SDG qualitative model of a qualitative reasoning leaving node by the state to increase or decrease the normal state).

Qualitative constraints mathematical description is: probability qualitative constraints, qualitative constraints facing the Piecewise function, fuzzy qualitative constraints, rough qualitative constraints[2].

Probability qualitative constraint is the use of a variety of qualitative information, and to introduce random elements, change the uncertain relationship to the conditions probability, so the formation of a Bayesian network.

Piecewise function qualitative constraint based on QSIM algorithm, made two major improvements: an increase of constraints to determine TF, access will be bound to a tree structure, scope limitations as to increase the assignment constraint $\mathrm{EQ}$, does not solve the function discontinuities.

Fuzzy qualitative constraint is a semi-quantitative qualitative constraint. In the fuzzy simulation algorithm, due to the introduction of fuzzy metric spaces, allows the use of fuzzy relations to represent the qualitative function constraints, the use of partial order information to establish quantitative functional dependencies between variables, Fuzzy qualitative simulation is mentioned in the constraint is not used to solve the unknown variable values, but to use the fuzzy relationship between the variables, testing the consistency of its value, is a form of qualitative constraints [3].

Rough qualitative constraint is a new concept that was proposed in this paper, it is mainly using the advantages of the rough set attribute reduction to reduce the results of qualitative reasoning and improve the binding efficiency.

\section{ThE CONSTRAINTS DESIGN OF ROUGH QUALITATIVE SOFTWARE}

A. The general idea of software design

Software Design: Qualitative reasoning results are connect to the database, according to rough set information table in the database fill in the data table , according to rough set attribute reduction algorithm, the data tables for all items in each category reduction, to identify important items which shows in the results window. 
Figure 1 is a general idea of the software design flow. B. software function modules

Software function modules include: Remove module, query module, add module, display module, table selection module, filter module, the main program, the output module. The filter module is the core module of the software, which is the rough set attribute reduction algorithm implementation modules.

Figure 2 is a software function block diagram.

\section{The EXAmPle of Qualitative CONSTRAINTS BASED ON ROUGH SET}

The overall function model of Materials Supply Support of was relatively simple qualitative simulation model, only 46 nodes, the statistics of the qualitative reasoning results was easier. If the model is to build complex, node number, artificially analyzed the results of reasoning was quite difficult. The use of rough set attribute reduction method, filtering out the factors of material supply capacity that influence is relatively small, quickly find an important factor in the material supply ability.

\section{A. The basis on Rough Qualitative Constraints}

The results collection of Material Supply Support qualitative reasoning are the set of uncertainty and certainty factors, such as "reserves" are uncertainties, and the "weather conditions" are uncertainties, so qualitative reasoning result set of Material Supply Support can be look as a rough set. According to the statistics of Material Supply Support qualitative simulation results, "abnormal reasons" of qualitative reasoning results, that is, the classified the degree of influence of Material Supply Support capacity, the establishment of Rough knowledge and information table of Material Supply Support, the use of Rough Set attribute reduction algorithm, reducing the overall function of the qualitative reasoning results of Material Supply Support.

\section{B. The realization of Material Supply Support using \\ Rough Qualitative Constraints}

Taking qualitative simulation experiment as example, integrated 6 times results of qualitative simulation experiment, are shown in Table 1, the rough knowledge and information table Material Supply Support.

The data of Material Supply Support in Rough knowledge information table were entered the rough qualitative constraint software to obtain three important reasons for non-normal (qualitative constraint programming provides the greatest influence on selecting only the top three factors), 3 unusual reasons, such as shown in Table 2.

After a rough qualitative constraint, it can be found that 3 "non-normal because" the path of the reasoning results of materials supply support qualitative simulation:

\section{bit state $(+) 2 \gg(-) 1 \gg(-) 33 \gg(-) 47$ \\ node name $\mathrm{PB} \gg \mathrm{BSC} \gg \mathrm{ISR} \gg \mathrm{FESC}$}

From the " material supply support command structure was to combat (FB)" Path analysis of all reasoning, the command function aspect of material supply support through all the features of other aspects of material supply support, that is the command of material raise, material reserve, material supply was exist in all the reasoning path.

$$
\begin{aligned}
& \text { bit state }(-) 5 \gg(-) 3 \gg(-) 1 \gg(-) 33\rangle)(-) 47 \\
& \text { node name } \mathbb{R} \quad \gg B C P \quad \gg B S C
\end{aligned}
$$

From the " roads severely damaged by enemy (RC)" reasoning path analysis, and all the path are related to command function, from the angle of local analysis that the "road conditions" should be more important in material transport, but through the Global Analysis of qualitative simulation Recognizing that "road conditions" on the impact to supply capacity of material supply support is relatively large.

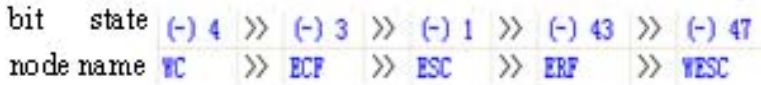

From the analysis of "weather conditions (WC)" reasoning path, all paths ultimately linked with the command, from the general perspective, "weather conditions" does feature material supply support, each link has an impact, but by qualitative simulation global analysis, it is recognized that the impact of " weather conditions " is closely related to the function of command.

In summary, the reasons for these three non-normal are relevant with material supply command function, which reflects the core position of material supply support command in wartime, and the adverse factor " that material supply support command structure was to combat " should be avoided.

\section{CONCLUSIONS}

The qualitative reasoning results of redundant and complex is restricted, which has been a hot and difficult problem in qualitative simulation. In this paper, integrated the superiority of rough set attribute reduction, this paper proposed a method of qualitative constraints, and designed the rough set constraint software. The results of material supply support in qualitative reasoning process are carried out experimental research, based on rough qualitative reduction of the constraints software. The results relying on many important results can really found through rough set constraints.

This is only a simple of rough qualitative constraints, testing the feasibility of this approach. In the next step, along this direction should be specific in-depth studies to enhance the applicability and universal of rough qualitative constraint.

\section{ACKNOWLEDGEMENTS}

Thanks for your help and advice to the paper.

\section{REFERENCES}

[1] LEI Shao-lan, SUN Cai-xin, ZHOU Lin etc. The application on attribute reduction in the space power load forecasting [J]. Chongqing University Transaction, 2004, 27 (3):85-88

[2] SONG Guang-ming, CAO Ji-ping, SONG Jian-she, HE Zhi-de. The resources optimal allocation of equipment maintenance support 
Based on Rough Set Theory [J]. Computer Engineering, 2007.2, 33 (4)
[3] ZHANG Wen-xiu, WU Wei-zhi, LIANG Ji-ye. Rough set theory and method $[\mathrm{M}]$, Beijing: Science Press, 2005.1

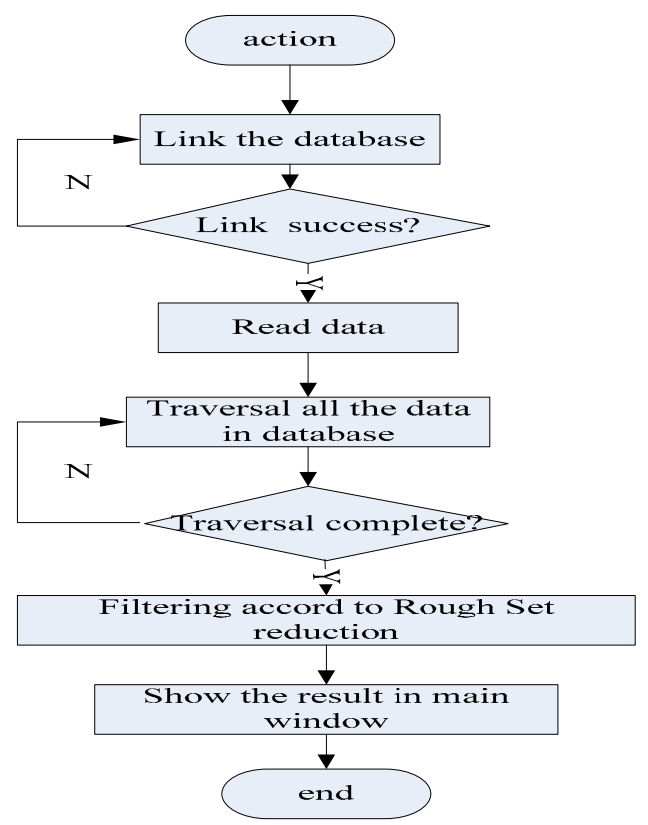

Figure 1 The general idea of the software flow chart

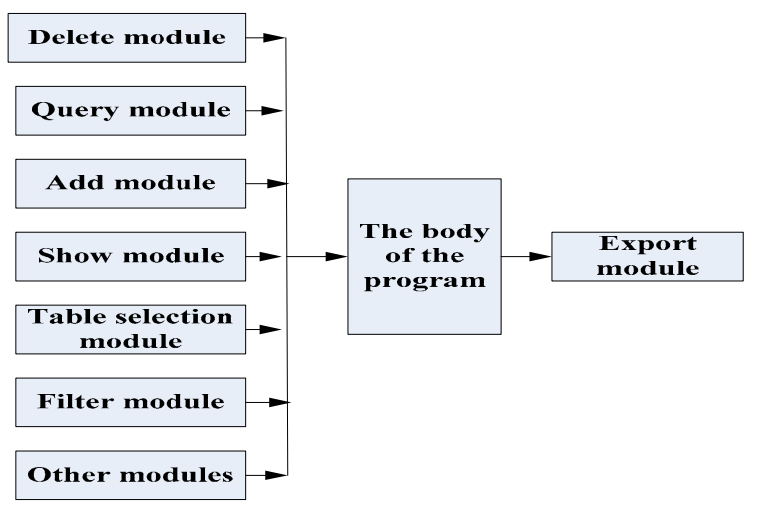

Figure 2 The overall design functional module

TABLE I. THE ROUGH KNOWLEDGE AND INFORMATION TABLE MATERIAL SUPPLY SUPPORT

\begin{tabular}{ccccccc}
\hline $\begin{array}{c}\text { Number of } \\
\text { experiment } \\
\text { s }\end{array}$ & $\begin{array}{c}\text { Supply } \\
\text { support } \\
\text { command } \\
\text { structure } \\
\text { (FB) }\end{array}$ & $\begin{array}{c}\text { road } \\
\text { conditions( } \\
\text { RC) }\end{array}$ & $\begin{array}{c}\text { storage } \\
\text { reserves } \\
\text { (SQ) }\end{array}$ & $\begin{array}{c}\text { Raising the } \\
\text { number } \\
\text { and } \\
\text { variety } \\
\text { (RDF) }\end{array}$ & $\begin{array}{c}\text { weather } \\
\text { conditio } \\
\text { ns(WC) }\end{array}$ & $\begin{array}{c}\text { Land } \\
\text { feature } \\
\text { (LC) }\end{array}$ \\
\hline 1 & 18 & 14 & 6 & 5 & 20 & 7 \\
2 & 14 & 14 & 5 & 5 & 20 & 7 \\
3 & 18 & 10 & 6 & 5 & 15 & 7 \\
4 & 18 & 14 & 3 & 5 & 20 & 5 \\
5 & 12 & 14 & 6 & 3 & 18 & 7 \\
6 & 18 & 10 & 4 & 5 & 18 & 7 \\
\hline
\end{tabular}


The 2nd International Conference on Computer Application and System Modeling (2012)

TABLE II. THE RESULTS OF QUALITATIVE SIMULATION AFTER ADDING QUALITATIVE CONSTRAINTS Bit Number Alphabet Denotation The Physical Meaning of the Letter

\begin{tabular}{ccc}
1 & FB & supply support command structure was to \\
& combat \\
2 & RC & roads severely damaged by enemy \\
5 & WC & climatic conditions \\
\hline
\end{tabular}

Published by Atlantis Press, Paris, France.

(C) the authors 0455 